\title{
Common fixed point theorem for generalized nonexpansive mappings on ordered orbitally complete metric spaces and application
}

\author{
Hemant Kumar Nashine and Ravi P. Agarwal
}

\begin{abstract}
We propose a common fixed point theorem for new notion of generalized nonexpansive mappings for two pairs of maps in an ordered orbitally complete metric space. To illustrate our result, we give throughout the paper two examples. Existence of solutions for certain system of functional equations arising in dynamic programming is also presented as application.
\end{abstract}

Mathematics Subject Classification (2010): 47H10, 54H 25 .

Keywords: Partially ordered set, nonexpansive mapping, orbitally complete metric space, common fixed point, weak annihilator, dominating maps, partially weakly increasing, weakly compatible.

\section{Introduction}

The significance of nonexpansive mappings was sketched, e.g., in 1980 by Bruck [8]. A nonexpansive mapping of a complete metric space need not have a fixed point (consider a translation operator $\mathcal{T}(x)=x+c$ in a Banach space). A fixed point of a nonexpansive mapping need not be unique (consider $\mathcal{T}=I$ ). To make certain the existence and/or uniqueness of fixed points we must assume supplementary conditions on $\mathcal{T}$ and/or the underlying space. Contraction mappings, isometries and orthogonal projection are all nonexpansive mappings. The study of nonexpansive mappings has been one of the main features in modern developments of fixed point theory-see for instance $[7,10]$. Browder et al. [7] proved that every nonexpansive mapping $\mathcal{T}$ from a convex bounded closed subset $C$ of a Hilbert space $\mathcal{X}$ into $C$ has a fixed point. There are also several interesting unsolved problems. The existence fixed point results for nonexpansive mapping is discussed in the paper $[10,11,14,27,30]$ and others.

In 1986, some near the beginning results in this direction were recognized in the papers of Turinici [31,32]; note that their starting points were the "amorphous" contributions in the area due to Matkowski $[15,16]$. These results have been revive 
by Ran and Reurings [26, Theorem 2.1], where they extended the Banach contraction principle in partially ordered sets with some applications to linear and nonlinear matrix equations. Subsequently, several authors obtained many fixed point theorems in the underlying space, see for more facts $[1,2,13,17,20,22,25,29]$ and the references cited therein. Recently, Nashine and Kadelburg [18] proved some results for two pairs of mapping for implicit type relations in ordered orbitally complete metric spaces.

We propose a new generalized nonexpansive mappings for two pairs of maps in ordered metric spaces and relevance to fixed point theorem on an ordered orbitally complete metric space. We furnish suitable examples to demonstrate the validity of the hypotheses of our result. Our result is extensions of the results of Ciric [10] and Nashine and Kadelburg [17] in the sense of considering two pairs of maps in an orbitally complete ordered metric space. In the final section, we apply the obtained result for proving the existence of solutions for certain system of functional equations arising in dynamic programming.

\section{Preliminaries}

We will bring into play the following notation and definitions. Consistent with Abbas et al. [1] the following definitions will be used all the way through the paper.

If $(\mathcal{X}, \preceq)$ is a partially ordered set then $x, y \in \mathcal{X}$ are called comparable if $x \preceq y$ or $y \preceq x$ holds. A subset $\mathcal{K}$ of $\mathcal{X}$ is said to be totally ordered if every two elements of $\mathcal{K}$ are comparable. If $\mathcal{T}: \mathcal{X} \rightarrow \mathcal{X}$ is such that, for $x, y \in \mathcal{X}, x \preceq y$ implies $\mathcal{T} x \preceq \mathcal{T} y$, then the mapping $\mathcal{T}$ is said to be nondecreasing.

Definition 2.1. Let $\mathcal{X}$ be a nonempty set. Then $(\mathcal{X}, d, \preceq)$ is called an ordered metric space if

(i) $(\mathcal{X}, d)$ is a metric space,

(ii) $(\mathcal{X}, \preceq)$ is a partially ordered set.

The space $(\mathcal{X}, d, \preceq)$ is called regular if the following hypothesis holds: if $\left\{z_{n}\right\}$ is a non-decreasing sequence in $\mathcal{X}$ with respect to $\preceq$ such that $z_{n} \rightarrow z \in \mathcal{X}$ as $n \rightarrow \infty$, then $z_{n} \preceq z$.

Definition 2.2. Let $(\mathcal{X}, \preceq)$ be a partially ordered set. A pair $(f, g)$ of selfmaps of $\mathcal{X}$ is said to be weakly increasing if $f x \preceq g f x$ and $g x \preceq f g x$ for all $x \in \mathcal{X}$.

Now we give a definition of partially weakly increasing pair of mappings.

Definition 2.3. Let $(\mathcal{X}, \preceq)$ be a partially ordered set and $f$ and $g$ be two selfmaps on $\mathcal{X}$. An ordered pair $(f, g)$ is said to be partially weakly increasing if $f x \preceq g f x$ for all $x \in \mathcal{X}$.

Note that a pair $(f, g)$ is weakly increasing if and only if ordered pair $(f, g)$ and $(g, f)$ are partially weakly increasing.

Following is an example of an ordered pair $(f, g)$ of selfmaps $f$ and $g$ which is partially weakly increasing but not weakly increasing. 
Example 2.4. Let $\mathcal{X}=[0,1]$ be endowed with usual ordering and $f, g: \mathcal{X} \rightarrow \mathcal{X}$ be defined by $f x=x^{2}$ and $g x=\sqrt{x}$. Clearly, $(f, g)$ is partially weakly increasing. But $g x=\sqrt{x} \neq x=f g x$ for $x \in(0,1)$ implies that $(g, f)$ is not partially weakly increasing.

Definition 2.5. Let $(\mathcal{X}, \preceq)$ be a partially ordered set. A mapping $f$ is a called weak annihilator of $g$ if $f g x \preceq x$ for all $x \in \mathcal{X}$.

Example 2.6. Let $\mathcal{X}=[0,1]$ be endowed with usual ordering and $f, g: \mathcal{X} \rightarrow \mathcal{X}$ be defined by $f x=x^{2}, g x=x^{3}$. Obviously, $f g x=x^{6} \leq x$ for all $x \in \mathcal{X}$. Thus $f$ is a weak annihilator of $g$.

Definition 2.7. Let $(\mathcal{X}, \preceq)$ be a partially ordered set. A mapping $f$ is called dominating if $x \preceq f x$ for each $x \in \mathcal{X}$.

Example 2.8. Let $\mathcal{X}=[0,1]$ be endowed with usual ordering and $f: \mathcal{X} \rightarrow \mathcal{X}$ be defined by $f x=x^{\frac{1}{3}}$. Since $x \leq x^{\frac{1}{3}}=f x$ for all $x \in \mathcal{X}$. Therefore $f$ is a dominating map.

Example 2.9. Let $\mathcal{X}=[0,4]$, endowed with usual ordering. Let $f, g: \mathcal{X} \rightarrow \mathcal{X}$ be defined by

$$
f x=\left\{\begin{array}{lll}
0, & \text { if } & x \in[0,1) \\
1, & \text { if } & x \in[1,3) \\
3, & \text { if } & x \in(3,4) \\
4, & \text { if } x=4
\end{array} \quad g x= \begin{cases}0, & \text { if } x=0 \\
1, & \text { if } x \in(0,1] \\
3, & \text { if } x \in(1,3] \\
4, & \text { otherwise }\end{cases}\right.
$$

The pair $(f, g)$ is partially weakly increasing and the dominating map $g$ is a weak annihilator of $f$.

Recall that the notion of orbitally complete metric space and orbitally continuous mapping were introduced by Ćirić in [9]. These definitions were extended to the case of two or three mappings by Sastry et al. in [28]. Some common fixed point results in this situation were obtained in $[12,19]$. We give now respective definitions for two pairs of mappings.

Definition 2.10. Let $\mathcal{A}, \mathcal{B}, \mathcal{S}, \mathcal{T}$ be four self-mappings defined on a metric space $(\mathcal{X}, d)$.

1. If for a point $x_{0} \in \mathcal{X}$, there exist sequences $\left\{x_{n}\right\}$ and $\left\{y_{n}\right\}$ in $\mathcal{X}$ such that

$$
y_{2 n-1}=\mathcal{A} x_{2 n-2}=\mathcal{T} x_{2 n-1}, \quad y_{2 n}=\mathcal{B} x_{2 n-1}=\mathcal{S} x_{2 n}, \quad \forall n \in \mathbb{N},
$$

then the set $\mathcal{O}\left(x_{0} ; \mathcal{A}, \mathcal{B}, \mathcal{S}, \mathcal{T}\right)=\left\{y_{n}: n=1,2, \ldots\right\}$ is called the orbit of $(\mathcal{A}, \mathcal{B}, \mathcal{S}, \mathcal{T})$ at $x_{0}$

2. The space $(\mathcal{X}, d)$ is said to be $(\mathcal{A}, \mathcal{B}, \mathcal{S}, \mathcal{T})$-orbitally complete at $x_{0}$ if every Cauchy sequence in $\mathcal{O}\left(x_{0} ; \mathcal{A}, \mathcal{B}, \mathcal{S}, \mathcal{T}\right)$ converges in $\mathcal{X}$.

3. The map $\mathcal{A}$ is said to be $(\mathcal{A}, \mathcal{B}, \mathcal{S}, \mathcal{T})$-orbitally continuous at $x_{0}$ if it is continuous on $\mathcal{O}\left(x_{0} ; \mathcal{A}, \mathcal{B}, \mathcal{S}, \mathcal{T}\right)$.

4. If $\mathcal{S}=\mathcal{T}$, we write $(\mathcal{A}, \mathcal{B}, \mathcal{S})$ in the previous definitions instead of $(\mathcal{A}, \mathcal{B}, \mathcal{S}, \mathcal{S})$, 


\section{Main results}

First, we introduce the notion of generalized nonexpansive mapping for four mappings in ordered metric spaces.

Definition 3.1. Let $(\mathcal{X}, d, \preceq)$ be an ordered metric space. We call two pairs of mappings $\mathcal{A}, \mathcal{B}, \mathcal{T}, \mathcal{S}: \mathcal{X} \rightarrow \mathcal{X}$ as generalized nonexpansive (of Ćirić type) if

$$
\begin{aligned}
d(\mathcal{A} x, \mathcal{B} y) \leq & a \max \left\{d(\mathcal{S} x, \mathcal{T} y), d(\mathcal{S} x, \mathcal{A} x), d(\mathcal{T} y, \mathcal{B} y), \frac{1}{2}[d(\mathcal{S} x, \mathcal{B} y)+d(\mathcal{T} y, \mathcal{A} x)]\right\} \\
& +b \max \{d(\mathcal{S} x, \mathcal{A} x), d(\mathcal{T} y, \mathcal{B} y)\}+c[d(\mathcal{S} x, \mathcal{B} y)+d(\mathcal{T} y, \mathcal{A} x)],
\end{aligned}
$$

holds for all comparable $x, y \in \mathcal{X}$, where $a \geq 0, b, c>0$ satisfy

$$
a+b+2 c=1 \text {. }
$$

Now, we state and prove our result.

Theorem 3.2. Let $(\mathcal{X}, d, \preceq)$ be a ordered metric space. Suppose that $\mathcal{T}, \mathcal{S}, \mathcal{A}, \mathcal{B}$ : $\mathcal{X} \rightarrow \mathcal{X}$ be given generalized nonexpansive mappings satisfying for every pair $x, y \in$ $\overline{\mathcal{O}\left(x_{0} ; \mathcal{A}, \mathcal{B}, \mathcal{S}, \mathcal{T}\right)}$ (for some $x_{0} \in \mathcal{X}$ ) such that $x$ and $y$ are comparable. We assume the following hypotheses:

(i) The space $(\mathcal{X}, d)$ is $(\mathcal{A}, \mathcal{B}, \mathcal{S}, \mathcal{T})$-orbitally complete at $x_{0}$;

(ii) $(\mathcal{T}, \mathcal{A})$ and $(\mathcal{S}, \mathcal{B})$ are partially weakly increasing on $\overline{\mathcal{O}\left(x_{0} ; \mathcal{A}, \mathcal{B}, \mathcal{S}, \mathcal{T}\right)}$;

(iii) $\mathcal{B} \mathcal{X} \subseteq \mathcal{S} \mathcal{X}$ and $\mathcal{A} \mathcal{X} \subseteq \mathcal{T} \mathcal{X}$;

(iv) $\mathcal{A}$ and $\mathcal{B}$ are dominating maps on $\overline{\mathcal{O}\left(x_{0} ; \mathcal{A}, \mathcal{B}, \mathcal{S}, \mathcal{T}\right)}$;

(v) $\mathcal{B}$ is a weak annihilator of $\mathcal{S}$ and $\mathcal{A}$ is a weak annihilator of $\mathcal{T}$ on $\overline{\mathcal{O}\left(x_{0} ; \mathcal{A}, \mathcal{B}, \mathcal{S}, \mathcal{T}\right)}$

(vi) For each nondecreasing sequence $\left\{x_{n}\right\}$ in $\mathcal{X}$, with $x_{n} \preceq y_{n}$ for all $n, y_{n} \rightarrow u$ implies that $x_{n} \preceq u$.

Assume either

(a) $(\mathcal{A}, \mathcal{S})$ is compatible, $\mathcal{A}$ or $\mathcal{S}$ is $(\mathcal{A}, \mathcal{B}, \mathcal{S}, \mathcal{T})$-orbitally continuous and $(\mathcal{B}, \mathcal{T})$ is weakly compatible, or

(b) $(\mathcal{B}, \mathcal{T})$ is compatible, $\mathcal{B}$ or $\mathcal{T}$ is $(\mathcal{A}, \mathcal{B}, \mathcal{S}, \mathcal{T})$-orbitally continuous and $(\mathcal{A}, \mathcal{S})$ is weakly compatible.

Then $\mathcal{A}, \mathcal{B}, \mathcal{S}$ and $\mathcal{T}$ have a common fixed point. Moreover, the set of common fixed points of $\mathcal{A}, \mathcal{B}, \mathcal{S}$ and $\mathcal{T}$ in $\overline{\mathcal{O}\left(x_{0} ; \mathcal{A}, \mathcal{B}, \mathcal{S}, \mathcal{T}\right)}$ is a singleton if and only if it is totally ordered.

Proof. Let $x_{0} \in \mathcal{X}$ be a point given in (i). Since $\mathcal{B X} \subseteq \mathcal{S X}$ and $\mathcal{A X} \subseteq \mathcal{T} \mathcal{X}$, we can consider sequences $\left\{x_{n}\right\}$ and $\left\{y_{n}\right\}$ in $\mathcal{X}$ given as in (2.1). By the given assumptions, $x_{2 n-2} \preceq \mathcal{A} x_{2 n-2}=\mathcal{T} x_{2 n-1} \preceq \mathcal{A T} x_{2 n-1} \preceq x_{2 n-1}$, and $x_{2 n-1} \preceq \mathcal{B} x_{2 n-1}=\mathcal{S} x_{2 n} \preceq$ $\mathcal{B S} x_{2 n} \preceq x_{2 n}$. Thus, for all $n \geq 0$, we have

$$
x_{n} \preceq x_{n+1} \text {. }
$$

Now we claim that $d\left(y_{n+1}, y_{n}\right) \leq d\left(y_{n}, y_{n-1}\right)$ for all $n \geq 1$. Suppose this is not true, that is, there exists $n_{0} \geq 1$ such that $d\left(y_{n_{0}+1}, y_{n_{0}}\right)>d\left(y_{n_{0}}, y_{n_{0}-1}\right)$. Now since $x_{n_{0}-1} \preceq x_{n_{0}}$, we can use the inequality (3.1) for these elements. 
Putting $x=x_{2 n_{0}+1}$ and $y=x_{2 n_{0}}$, from (3.2) and the considered contraction (3.1), we have

$$
\begin{aligned}
& d\left(y_{2 n_{0}+2}, y_{2 n_{0}+1}\right)=d\left(\mathcal{A} x_{2 n_{0}+1}, \mathcal{B} x_{2 n_{0}}\right) \\
& \leq \\
& \quad a \max \left\{\begin{array}{c}
d\left(\mathcal{S} x_{2 n_{0}+1}, \mathcal{T} x_{2 n_{0}}\right), d\left(\mathcal{S} x_{2 n_{0}+1}, \mathcal{A} x_{2 n_{0}+1}\right), d\left(\mathcal{T} x_{2 n_{0}}, \mathcal{B} x_{2 n_{0}}\right), \\
\frac{1}{2}\left[d\left(\mathcal{S} x_{2 n_{0}+1}, \mathcal{B} x_{2 n_{0}}\right)+d\left(\mathcal{T} x_{2 n_{0}}, \mathcal{A} x_{2 n_{0}+1}\right)\right]
\end{array}\right\} \\
& \quad+b \max \left\{d\left(\mathcal{S} x_{2 n_{0}+1}, \mathcal{A} x_{2 n_{0}+1}\right), d\left(\mathcal{T} x_{2 n_{0}}, \mathcal{B} x_{2 n_{0}}\right)\right\} \\
& \quad+c\left[d\left(\mathcal{S} x_{2 n_{0}+1}, \mathcal{B} x_{2 n_{0}}\right)+d\left(\mathcal{T} x_{2 n_{0}}, \mathcal{A} x_{2 n_{0}+1}\right)\right] \\
& = \\
& \quad a \max \left\{d\left(y_{2 n_{0}+1}, y_{2 n_{0}}\right), \frac{1}{2} d\left(y_{2 n_{0}}, y_{2 n_{0}+2}\right)\right\} \\
& \quad+b \max \left\{d\left(y_{2 n_{0}+1}, y_{2 n_{0}+2}\right), d\left(y_{2 n_{0}}, y_{2 n_{0}+1}\right)\right\}+c d\left(y_{2 n_{0}}, y_{2 n_{0}+2}\right) .
\end{aligned}
$$

Using a triangular inequality, we have

$$
\frac{1}{2} d\left(y_{2 n_{0}}, y_{2 n_{0}+2}\right) \leq \frac{1}{2}\left(d\left(y_{2 n_{0}}, y_{2 n_{0}+1}\right)+d\left(y_{2 n_{0}+1}, y_{2 n_{0}+2}\right)\right)<d\left(y_{2 n_{0}+1}, y_{2 n_{0}+2}\right) \text {. }
$$

Since $c>0$, this implies that

$$
\begin{aligned}
d\left(y_{2 n_{0}+2}, y_{2 n_{0}+1}\right) & <(a+b) d\left(y_{2 n_{0}+1}, y_{2 n_{0}+2}\right)+2 c d\left(y_{2 n_{0}+1}, y_{2 n_{0}+2}\right) \\
& =(a+b+2 c) d\left(y_{2 n_{0}+1}, y_{2 n_{0}+2}\right)=d\left(y_{2 n_{0}+2}, y_{2 n_{0}+1}\right)
\end{aligned}
$$

a contradiction. Thus $d\left(\mathcal{A} x_{2 n+1}, \mathcal{B} x_{2 n+1}\right) \leq d\left(\mathcal{A} x_{2 n}, \mathcal{B} x_{2 n}\right)$. Hence

$$
d\left(\mathcal{A} x_{n+1}, \mathcal{B} x_{n+1}\right) \leq d\left(\mathcal{A} x_{0}, \mathcal{B} x_{0}\right), \text { for all positive integers } n .
$$

Using (3.1) and (3.3) and triangle inequality, we have

$$
\begin{aligned}
d & \left(y_{2 n-1}, \mathcal{B} x_{2 n}\right)=d\left(\mathcal{A} x_{2 n-2}, \mathcal{B} x_{2 n}\right) \\
\leq & a \max \left\{\begin{array}{c}
d\left(\mathcal{S} x_{2 n-2}, \mathcal{T} x_{2 n}\right), d\left(\mathcal{S} x_{2 n-2}, \mathcal{A} x_{2 n-2}\right), d\left(\mathcal{T} x_{2 n}, \mathcal{B} x_{2 n}\right) \\
\frac{1}{2}\left[d\left(\mathcal{S} x_{2 n-2}, \mathcal{B} x_{2 n}\right)+d\left(\mathcal{T} x_{2 n}, \mathcal{A} x_{2 n-2}\right)\right]
\end{array}\right\} \\
& +b \max \left\{d\left(\mathcal{S} x_{2 n-2}, \mathcal{A} x_{2 n-2}\right), d\left(\mathcal{T} x_{2 n}, \mathcal{B} x_{2 n}\right)\right\} \\
& +c\left[d\left(\mathcal{S} x_{2 n-2}, \mathcal{B} x_{2 n}\right)+d\left(\mathcal{T} x_{2 n}, \mathcal{A} x_{2 n-2}\right)\right] \\
= & a \max \left\{\begin{array}{c}
d\left(y_{2 n-2}, y_{2 n}\right), d\left(y_{2 n-2}, y_{2 n-1}\right), d\left(y_{2 n}, y_{2 n+1}\right), \\
\frac{1}{2}\left[d\left(y_{2 n-2}, y_{2 n+1}\right)+d\left(y_{2 n}, y_{2 n-1}\right)\right]
\end{array}\right\} \\
& +b \max \left\{d\left(y_{2 n-2}, y_{2 n-1}\right), d\left(y_{2 n}, y_{2 n+1}\right)\right\}+c\left[d\left(y_{2 n-2}, y_{2 n+1}\right)+d\left(y_{2 n}, y_{2 n-1}\right)\right] .
\end{aligned}
$$

From (3.3) and the triangle inequality we get

$$
\begin{aligned}
& \frac{1}{2}\left[d\left(y_{2 n-2}, y_{2 n+1}\right)+d\left(y_{2 n}, y_{2 n-1}\right)\right] \\
& \left.\leq \frac{1}{2}\left[d\left(y_{2 n-2}, y_{2 n-1}\right)+d\left(y_{2 n}, y_{2 n-1}\right)+d\left(y_{2 n}, y_{2 n+1}\right)+d\left(y_{2 n}, y_{2 n-1}\right)\right)\right] \\
& \leq 2 d\left(y_{2 n-2}, y_{2 n-1}\right) .
\end{aligned}
$$

Substituting (3.6) in (3.4), we have

$$
\begin{aligned}
d\left(y_{2 n-1}, \mathcal{B} x_{2 n}\right) & \leq 2 a d\left(y_{2 n-2}, y_{2 n-1}\right)+b d\left(y_{2 n-1}, y_{2 n-1}\right)+4 c d\left(y_{2 n-2}, y_{2 n-1}\right) \\
& =(2 a+b+4 c) d\left(y_{2 n-2}, y_{2 n-1}\right) .
\end{aligned}
$$

Hence $d\left(y_{2 n-1}, \mathcal{B} x_{2 n}\right)=(2-b) d\left(y_{2 n-2}, y_{2 n-1}\right)$. 
From (3.1), (3.3) and (3.6), we have

$$
\begin{aligned}
& d\left.y_{2 n}, \mathcal{B} x_{2 n}\right)=d\left(\mathcal{A} x_{2 n-1}, \mathcal{B} x_{2 n}\right) \\
& \leq a \max \left\{\begin{array}{c}
\left.d\left(\mathcal{S} x_{2 n-1}, \mathcal{T} x_{2 n}\right), d\left(\mathcal{S} x_{2 n-1}, \mathcal{A} x_{2 n-1}\right), d\left(\mathcal{T} x_{2 n}, \mathcal{B} x_{2 n}\right), c c\right\} \\
\frac{1}{2}\left[d\left(\mathcal{S} x_{2 n-1}, \mathcal{B} x_{2 n}\right)+d\left(\mathcal{T} x_{2 n}, \mathcal{A} x_{2 n-1}\right)\right]
\end{array}\right. \\
& \quad+b \max \left\{d\left(\mathcal{S} x_{2 n-1}, \mathcal{A} x_{2 n-1}\right), d\left(\mathcal{T} x_{2 n}, \mathcal{B} x_{2 n}\right)\right\} \\
& \quad+c\left[d\left(\mathcal{S} x_{2 n-1}, \mathcal{B} x_{2 n}\right)+d\left(\mathcal{T} x_{2 n}, \mathcal{A} x_{2 n-1}\right)\right] \\
&= a \max \left\{d\left(y_{2 n-1}, y_{2 n}\right), d\left(y_{2 n-1}, y_{2 n}\right), d\left(y_{2 n}, y_{2 n+1}\right), \frac{1}{2}\left[d\left(y_{2 n-1}, y_{2 n+1}\right)+d\left(y_{2 n}, y_{2 n}\right)\right]\right\} \\
&+b \max \left\{d\left(y_{2 n-1}, y_{2 n}\right), d\left(y_{2 n}, y_{2 n+1}\right)\right\}+c\left[d\left(y_{2 n-1}, y_{2 n+1}\right)+d\left(y_{2 n}, y_{2 n}\right)\right] \\
& \leq a d\left(y_{2 n-2}, y_{2 n-1}\right)+b d\left(y_{2 n-2}, y_{2 n-1}\right)+c(2-b) d\left(y_{2 n-2}, y_{2 n-1}\right)
\end{aligned}
$$

and hence

$$
d\left(y_{2 n}, \mathcal{B} x_{2 n}\right)=(1-b c) d\left(y_{2 n-2}, y_{2 n-1}\right) .
$$

Proceeding in this manner we obtain

$$
d\left(y_{2 n}, \mathcal{B} x_{2 n}\right) \leq(1-b c)^{\left[\frac{n}{2}\right]} d\left(y_{0}, y_{1}\right)
$$

for all $n=1,2, \ldots$, where $\frac{n}{2}$ denotes the greatest integer not exceeding $\frac{n}{2}$. Since $1-b c<1$, from (3.7), we conclude that $\left\{y_{n}\right\}$ is a Cauchy sequence.

Finally, we prove the existence of a common fixed point of the four mappings $\mathcal{A}, \mathcal{B}, \mathcal{S}$ and $\mathcal{T}$.

Since $\left\{y_{n}\right\}$ is a Cauchy sequence, defined by $(2.1)$ in an $(\mathcal{A}, \mathcal{B}, \mathcal{S}, \mathcal{T})$-orbitally complete metric space $(\mathcal{X}, d)$, there exists a point $z$ in $\mathcal{X}$, such that $y_{n}$ converges to $z$. Therefore,

$$
y_{2 n+1}=\mathcal{T} x_{2 n+1}=\mathcal{A} x_{2 n} \rightarrow z \text { as } n \rightarrow \infty
$$

and

$$
y_{2 n+2}=\mathcal{S} x_{2 n+2}=\mathcal{B} x_{2 n+1} \rightarrow z \text { as } n \rightarrow \infty .
$$

Suppose that (a) holds. Since $(\mathcal{A}, \mathcal{S})$ is compatible, we have

$$
\lim _{n \rightarrow \infty} \mathcal{A S} x_{2 n+2}=\lim _{n \rightarrow \infty} \mathcal{S} \mathcal{A} x_{2 n+2}=\mathcal{S} z
$$

Also, $x_{2 n+1} \preceq \mathcal{B} x_{2 n+1}=\mathcal{S} x_{2 n+2}$. Now

$$
\begin{aligned}
& d\left(\mathcal{A S} x_{2 n+2}, \mathcal{B} x_{2 n+1}\right) \\
& \leq a \max \left\{\begin{array}{c}
d\left(\mathcal{S} x_{2 n+2}, \mathcal{T} x_{2 n+1}\right), d\left(\mathcal{S S} x_{2 n+2}, \mathcal{A S} x_{2 n+2}\right), d\left(\mathcal{T} x_{2 n+1}, \mathcal{B} x_{2 n+1}\right), \\
\frac{1}{2}\left[d\left(\mathcal{S S} x_{2 n+2}, \mathcal{B} x_{2 n+1}\right)+d\left(\mathcal{T} x_{2 n+1}, \mathcal{A S} x_{2 n+2}\right)\right]
\end{array}\right\} \\
& \quad+b \max \left\{d\left(\mathcal{S S} x_{2 n+2}, \mathcal{A S} x_{2 n+2}\right), d\left(\mathcal{T} x_{2 n+1}, \mathcal{B} x_{2 n+1}\right)\right\} \\
& +c\left[d\left(\mathcal{S} \mathcal{S} x_{2 n+2}, \mathcal{B} x_{2 n+1}\right)+d\left(\mathcal{T} x_{2 n+1}, \mathcal{A S} x_{2 n+2}\right)\right] .
\end{aligned}
$$

Assume that $\mathcal{S}$ is $(\mathcal{A}, \mathcal{B}, \mathcal{S}, \mathcal{T})$-orbitally continuous. Passing to the limit as $n \rightarrow \infty$, we obtain

$$
\begin{aligned}
d(\mathcal{S} z, z) & \leq a \max \left\{d(\mathcal{S} z, z), 0,0, \frac{1}{2}[d(\mathcal{S} z, z)+d(z, \mathcal{S} z)]\right\} \\
& +b \max \{0,0\}+c[d(\mathcal{S} z, z)+d(z, \mathcal{S} z)] \\
& \leq(a+2 c) d(\mathcal{S} z, z)=(1-b) d(\mathcal{S} z, z)
\end{aligned}
$$


Since $b>0$ and $(1-b)<1$, this implies that

$$
\mathcal{S} z=z .
$$

Now, $x_{2 n+1} \preceq \mathcal{B} x_{2 n+1}$ and $\mathcal{B} x_{2 n+1} \rightarrow z$ as $n \rightarrow+\infty$, so by assumption we have $x_{2 n+1} \preceq z$ and $(3.1)$ becomes

$$
\begin{aligned}
& d\left(\mathcal{A} z, \mathcal{B} x_{2 n+1}\right) \leq a \max \left\{\begin{array}{c}
d\left(\mathcal{S} z, \mathcal{T} x_{2 n+1}\right), d(\mathcal{S} z, \mathcal{A} z), d\left(\mathcal{T} x_{2 n+1}, \mathcal{B} x_{2 n+1}\right), \\
\frac{1}{2}\left[d\left(\mathcal{S} z, \mathcal{B} x_{2 n+1}\right)+d\left(\mathcal{T} x_{2 n+1}, \mathcal{A} z\right)\right]
\end{array}\right\} \\
&+b \max \left\{d(\mathcal{S} z, \mathcal{A} z), d\left(\mathcal{T} x_{2 n+1}, \mathcal{B} x_{2 n+1}\right)\right\} \\
&+c\left[d\left(\mathcal{S} z, \mathcal{B} x_{2 n+1}\right)+d\left(\mathcal{T} x_{2 n+1}, \mathcal{A} z\right)\right]
\end{aligned}
$$

Passing to the limit $n \rightarrow+\infty$ in the above inequality and using (3.10),

$$
\begin{aligned}
d(\mathcal{A} z, z) \leq & a \max \left\{d(\mathcal{S} z, z), d(\mathcal{S} z, \mathcal{A} z), d(z, z), \frac{1}{2}[d(\mathcal{S} z, z)+d(z, \mathcal{A} z)]\right\} \\
& +b \max \{d(\mathcal{S} z, \mathcal{A} z), d(z, z)\}+c[d(\mathcal{S} z, z)+d(z, \mathcal{A} z)] \\
= & (a+b+c) d(z, \mathcal{A} z) .
\end{aligned}
$$

Since $a, b, c>0$ and $(a+b+c)<1$, this implies that

$$
\mathcal{A} z=z \text {. }
$$

Since $\mathcal{A}(\mathcal{X}) \subseteq \mathcal{T}(\mathcal{X})$, there exists a point $\omega \in \mathcal{X}$ such that $\mathcal{A} z=\mathcal{T} \omega$. Suppose that $\mathcal{T} \omega \neq \mathcal{B} \omega$. Since $z \preceq \mathcal{A} z=\mathcal{T} \omega \preceq \mathcal{A} \mathcal{T} \omega \preceq \omega$ implies $z \preceq \omega$. From (3.1), we obtain

$$
\begin{aligned}
& d(\mathcal{T} \omega, \mathcal{B} \omega)=d(\mathcal{A} z, \mathcal{B} \omega) \\
& \leq a \max \left\{d(\mathcal{S} z, \mathcal{T} \omega), d(\mathcal{S} z, \mathcal{A} z), d(\mathcal{T} \omega, \mathcal{B} \omega), \frac{1}{2}[d(\mathcal{S} z, \mathcal{B} \omega)+d(\mathcal{T} \omega, \mathcal{A} z)]\right\} \\
& \quad+b \max \{d(\mathcal{S} z, \mathcal{A} z), d(\mathcal{T} \omega, \mathcal{B} \omega)\}+c[d(\mathcal{S} z, \mathcal{B} \omega)+d(\mathcal{T} \omega, \mathcal{A} z)] \\
& \left.\leq a \max \left\{d(z, \mathcal{T} \omega), 0, d(\mathcal{T} \omega, \mathcal{B} \omega), \frac{1}{2} d(z, \mathcal{B} \omega)\right\}+b \max \{0, d(\mathcal{T} \omega, \mathcal{B} \omega)\}+c d(z, \mathcal{B} \omega)\right] \\
& =(a+b+c) d(\mathcal{T} \omega, \mathcal{B} \omega)
\end{aligned}
$$

contradiction to the state $a+b+2 c=1$. Hence, we get

$$
\mathcal{T} \omega=\mathcal{B} \omega \text {. }
$$

Since $\mathcal{B}$ and $\mathcal{T}$ are weakly compatible, $\mathcal{B} z=\mathcal{B} \mathcal{A} z=\mathcal{B} \mathcal{T} w=\mathcal{T} \mathcal{B} w=\mathcal{T} \mathcal{A} z=\mathcal{T} z$. Thus $z$ is a coincidence point of $\mathcal{B}$ and $\mathcal{T}$.

Now, since $x_{2 n} \preceq \mathcal{A} x_{2 n}$ and $\mathcal{A} x_{2 n} \rightarrow z$ as $n \rightarrow \infty$, implies that $x_{2 n} \preceq z$, from (3.1)

$$
\begin{aligned}
& d\left(\mathcal{A} x_{2 n}, \mathcal{B} z\right) \\
& \quad \leq a \max \left\{\begin{array}{c}
d\left(\mathcal{S} x_{2 n}, \mathcal{T} z\right), d\left(\mathcal{S} x_{2 n}, \mathcal{A} x_{2 n}\right), d(\mathcal{T} z, \mathcal{B} z) \\
\frac{1}{2}\left[d\left(\mathcal{S} x_{2 n}, \mathcal{B} z\right)+d\left(\mathcal{T} z, \mathcal{A} x_{2 n}\right)\right]
\end{array}\right\} \\
& \quad+b \max \left\{d\left(\mathcal{S} x_{2 n}, \mathcal{A} x_{2 n}\right), d(\mathcal{T} z, \mathcal{B} z)\right\}+c\left[d\left(\mathcal{S} x_{2 n}, \mathcal{B} z\right)+d\left(\mathcal{T} z, \mathcal{A} x_{2 n}\right)\right]
\end{aligned}
$$


Passing to the limit as $n \rightarrow+\infty$, we have

$$
\begin{aligned}
d(z, \mathcal{B} z) \leq & a \max \left\{d(z, \mathcal{B} z), 0,0, \frac{1}{2}[d(z, \mathcal{B} z)+d(\mathcal{B} z, z)]\right\} \\
& +b \max \{0,0)\}+c[d(z, \mathcal{B} z)+d(\mathcal{B} z, z)] \\
= & (a+2 c) d(z, \mathcal{B} z)=(1-b) d(z, \mathcal{B} z) .
\end{aligned}
$$

Since $b>0$ and $(1-b)<1$, which gives that

$$
z=\mathcal{B} z \text {. }
$$

Therefore, $\mathcal{A} z=\mathcal{B} z=\mathcal{S} z=\mathcal{T} z=z$, so $z$ is a common fixed point of $\mathcal{A}, \mathcal{B}, \mathcal{S}$ and $\mathcal{T}$.

The proof is similar when $\mathcal{A}$ is orbitally continuous.

Similarly, the result follows when $(b)$ holds.

Now, suppose that the set of common fixed points of $\mathcal{S}, \mathcal{T}, \mathcal{A}$ and $\mathcal{B}$ is totally ordered. We claim that there is a unique common fixed point of $\mathcal{A}, \mathcal{B}, \mathcal{S}$ and $\mathcal{T}$. Assume on contrary that $\mathcal{S} u=\mathcal{T} u=\mathcal{A} u=\mathcal{B} u=u$ and $\mathcal{S} \vartheta=\mathcal{T} \vartheta=\mathcal{A} \vartheta=\mathcal{B} \vartheta=\vartheta$ but $u \neq \vartheta$. By supposition, we can replace $x$ by $u$ and $y$ by $\vartheta$ in (3.1) to obtain

$$
\begin{aligned}
d(u, \vartheta)= & d(\mathcal{A} u, \mathcal{B} \vartheta) \\
\leq & a \max \left\{d(\mathcal{S} u, \mathcal{T} \vartheta), d(\mathcal{S} u, \mathcal{A} u), d(\mathcal{T} \vartheta, \mathcal{B} \vartheta), \frac{1}{2}[d(\mathcal{S} u, \mathcal{B} \vartheta)+d(\mathcal{T} \vartheta, \mathcal{A} u)]\right\} \\
& \quad+b \max \{d(\mathcal{S} u, \mathcal{A} u), d(\mathcal{T} \vartheta, \mathcal{B} \vartheta)\}+c[d(\mathcal{S} u, \mathcal{B} \vartheta)+d(\mathcal{T} \vartheta, \mathcal{A} u)] \\
= & (a+2 c) d(u, \vartheta)=(1-b) d(u, \vartheta) .
\end{aligned}
$$

Since $b>0$, this implies that $u=\vartheta$.

Conversely, if $\mathcal{A}, \mathcal{B}, \mathcal{S}$ and $\mathcal{T}$ have only one common fixed point, then the set of common fixed point of $\mathcal{S}, \mathcal{T}, \mathcal{A}$ and $\mathcal{B}$ being singleton is totally ordered. This completes the proof.

As consequence of Theorem 3.2, we may state the following corollary.

Corollary 3.3. Let $(\mathcal{X}, d, \preceq)$ be an ordered metric space. Let $\mathcal{A}, \mathcal{B}, \mathcal{S}: \mathcal{X} \rightarrow \mathcal{X}$ be given mappings satisfying for every pair $x, y \in \overline{\mathcal{O}\left(x_{0} ; \mathcal{A}, \mathcal{B}, \mathcal{S}\right)}$ (for some $x_{0} \in \mathcal{X}$ ) such that $x$ and $y$ are comparable,

$$
\begin{aligned}
d(\mathcal{A} x, \mathcal{B} y) \leq & a \max \left\{d(\mathcal{S} x, \mathcal{S} y), d(\mathcal{S} x, \mathcal{A} x), d(\mathcal{S} y, \mathcal{B} y), \frac{1}{2}[d(\mathcal{S} x, \mathcal{B} y)+d(\mathcal{S} y, \mathcal{A} x)]\right\} \\
+ & b \max \{d(\mathcal{S} x, \mathcal{A} x), d(\mathcal{S} y, \mathcal{B} y)\}+c[d(\mathcal{S} x, \mathcal{B} y)+d(\mathcal{S} y, \mathcal{A} x)]
\end{aligned}
$$

holds for all comparable $x, y \in \mathcal{X}$, where $a \geq 0, b, c>0$ satisfy

$$
a+b+2 c=1 \text {. }
$$

The mappings $\mathcal{A}, \mathcal{B}, \mathcal{S}$ satisfy (i)-(vi) and (a) (or (b)) of Theorem 3.2. Then $\mathcal{A}, \mathcal{B}$ and $\mathcal{S}$ have a common fixed point. Moreover, the set of common fixed points of $\mathcal{A}, \mathcal{B}$ and $\mathcal{S}$ in $\overline{\mathcal{O}\left(x_{0} ; \mathcal{A}, \mathcal{B}, \mathcal{S}\right)}$ is a singleton if and only if it is totally ordered.

Proof. It follows by taking $\mathcal{T}=\mathcal{S}$ in (3.1) and Theorem 3.2. 
By choosing $\mathcal{A}, \mathcal{B}, \mathcal{S}$ and $\mathcal{T}$ suitably in Theorem 3.2, we can deduce some corollaries for a pair as well as for a triple of self mappings.

In what follows, we support the result of Theorem 3.2 by examples.

Following example is inspired by [18].

Example 3.4. Let $\mathcal{X}=[0,+\infty)$ be equipped with the standard metric and order. Consider the mappings $\mathcal{A}, \mathcal{B}, \mathcal{S}, \mathcal{T}: \mathcal{X} \rightarrow \mathcal{X}$ given by

$$
\begin{aligned}
& \mathcal{A} x= \begin{cases}\frac{1+x}{2}, & 0 \leq x \leq 1 \\
4 x-3, & x>1,\end{cases} \\
& \mathcal{S} x= \begin{cases}0, & \mathcal{B} x= \begin{cases}\frac{2+x}{3}, & 0 \leq x \leq 1 \\
3 x-2, & x>1,\end{cases} \\
6 x-5, & x>\frac{5}{6},\end{cases}
\end{aligned}
$$

Conditions (i)-(vi) and (a) (or (b)) of Theorem 3.2 are easy to check for $x_{0}=\frac{5}{6}$. Then $\mathcal{O}\left(x_{0} ; \mathcal{A}, \mathcal{B}, \mathcal{S}, \mathcal{T}\right) \subset\left[\frac{5}{6}, 1\right]$

Note, though, that conditions (iii) and (v) are not satisfied on the entire space $\mathcal{X}$.

At present we will prove that condition (3.1) is fulfilled with $x_{0}=\frac{5}{6}, a=$ $\frac{2}{5}, b=\frac{1}{5}, c=\frac{1}{5}$. Then $a, b, c$ undoubtedly accomplish all conditions, in particular $a+b+2 c=1$.

Take $x, y \in \mathcal{O}\left(x_{0} ; \mathcal{A}, \mathcal{B}, \mathcal{S}, \mathcal{T}\right) \subset\left[0, \frac{5}{6}\right]$. Then $(3.1)$ converts to

$$
\begin{aligned}
\left|\frac{1+x}{2}-\frac{2+y}{3}\right| \leq & \frac{2}{5} \max \left\{\begin{array}{c}
|6 x-5 y-1|, \frac{11(1-x)}{2}, \frac{17(y-1)}{3}, \\
\frac{1}{2}\left[\left|6 x-5-\frac{2+y}{3}\right|+\left|5 y-4-\frac{1+x}{2}\right|\right]
\end{array}\right\} \\
& +\frac{1}{5} \max \left\{\frac{11(1-x)}{2}, \frac{14(1-y)}{3}\right\} \\
& +\frac{1}{5}\left[\left|6 x-5-\frac{2+y}{3}\right|+\left|5 y-4-\frac{1+x}{2}\right|\right] .
\end{aligned}
$$

By means of the replacement $x=1-\xi, y=1-\xi t, 0 \leq \xi \leq 1, t \geq 0$, the preceding inequality turn into

$$
\begin{aligned}
&\left|\frac{t}{3}-\frac{1}{2}\right| \leq \frac{2}{5} \max \left\{|5 t-6|, \frac{11}{2}, \frac{17 t}{3}, \frac{1}{2}\left[\left|\frac{t}{3}-6\right|+\left|5 t-\frac{1}{2}\right|\right]\right\} \\
&+\frac{1}{5} \max \left\{\frac{11}{2}, \frac{14 t}{3}\right\}+\frac{1}{5}\left[\left|\frac{t}{3}-6\right|+\left|5 t-\frac{1}{2}\right|\right]
\end{aligned}
$$

and can be tested out by argument on feasible values of $t \geq 0$. It is remark that condition (3.1) does not hold exterior of $\mathcal{O}\left(x_{0} ; \mathcal{A}, \mathcal{B}, \mathcal{S}, \mathcal{T}\right)$. For instance, it is adequate to take $x=2$ and $y=3$.

Thus, $\mathcal{A}, \mathcal{B}, \mathcal{S}, \mathcal{T}$ have a (unique) common fixed point (which is $z=1$ ).

Following is the another example, inspired by [23, 18].

Example 3.5. Let $\mathcal{X}=[0, \infty)$ with the usual distance and define an ordering $\preceq$ on $\mathcal{X}$ as follows:

$$
x \preceq y \Longleftrightarrow x=y \text { or }(x, y \in[0,1] \text { and } y \leq x) \text {. }
$$


Define $\mathcal{A}, \mathcal{B}, \mathcal{S}, \mathcal{T}: \mathcal{X} \rightarrow \mathcal{X}$ by

$$
\begin{gathered}
\mathcal{A} x=\left\{\begin{array}{ll}
\ln \left(\frac{x}{2}+1\right), & 0 \leq x \leq 1 \\
3 x, & x>1,
\end{array} \quad \mathcal{B} x= \begin{cases}\ln \left(\frac{x}{3}+1\right), & 0 \leq x \leq 1 \\
2 x, & x>1,\end{cases} \right. \\
\mathcal{S} x=\frac{e^{6 x}-1}{6}, \quad \mathcal{T} x=\frac{e^{4 x}-1}{6} .
\end{gathered}
$$

Take $x_{0}=1$.

Then $\mathcal{O}\left(x_{0} ; \mathcal{A}, \mathcal{B}, \mathcal{S}, \mathcal{T}\right) \subset(0,1)$ and $\overline{\mathcal{O}\left(x_{0} ; \mathcal{A}, \mathcal{B}, \mathcal{S}, \mathcal{T}\right)}=\mathcal{O}\left(x_{0} ; \mathcal{A}, \mathcal{B}, \mathcal{S}, \mathcal{T}\right) \cup\{0\}$.

It is easy to prove all conditions of Theorem 3.2 from (i)-(vi) and (a)-(b) along with condition (3.1) satisfy and 0 is the unique common fixed point of $\mathcal{A}, \mathcal{B}, \mathcal{S}$ and $\mathcal{T}$ in $\overline{\mathcal{O}\left(x_{0} ; \mathcal{A}, \mathcal{B}, \mathcal{S}, \mathcal{T}\right)}$.

It is observed that the conditions of Theorem 3.2 do not hold on the complete space $\mathcal{X}$.

\section{Application to functional equations arising in dynamic programming}

The fundamental shape of the functional equation of dynamic programming is given by Bellman and Lee [5] as follows:

$$
q(x)=\operatorname{opt}_{y \in \mathcal{D}}\{G(x, y, q(\tau(x, y)))\}, \quad x \in W,
$$

where $\tau: W \times \mathcal{D} \rightarrow W, G: W \times \mathcal{D} \times \mathbb{R} \rightarrow \mathbb{R}$ are mappings, while $W \subseteq U$ is a state space, $\mathcal{D} \subseteq V$ is a decision space, and $U, V$ are Banach spaces. Here $x$ and $y$ represent the state and decision vectors respectively, $\tau$ represents the transformation of the process and $q(x)$ represents the optimal return with initial state $x$ (where opt denotes max or min).

Subsequently a lot of work have been done in this trend and existence and uniqueness outcome have been attained for solutions and common solutions of some functional equations, as well as systems of functional equations in dynamic programming with the use of fixed point results. For details see $[6,24]$ and the references therein.

Let $\mathcal{X}=B(W)$ be the set of all bounded real-valued functions on $W$. According to the ordinary addition of functions and scalar multiplication, and with the norm $\|\cdot\|_{\infty}$ given by

$$
\|h\|_{\infty}=\sup _{x \in W}|h(x)| \text { for all } h \in \mathcal{X},
$$

we have that $\left(\mathcal{X},\|\cdot\|_{\infty}\right)$ is a Banach space and the respective convergence is uniform. In fact, the distance in $\mathcal{X}$ is given by

$$
d_{\infty}(u, v)=\sup _{x \in W}|u(x)-v(x)| \text { for all } u, v \in \mathcal{X} .
$$

Therefore, if we consider a Cauchy sequence $\left\{h_{n}\right\}$ in $\mathcal{X}$, then it converges uniformly to a function, say $h^{*}$, that is bounded. Therefore $h^{*} \in \mathcal{X}$.

Let $\sqsubseteq$ be the partial order relation on $\mathcal{X}$ defined by

$$
x \sqsubseteq y \text { if and only if } x(t) \leq y(t) \text { for any } t \in W .
$$


Then $(\mathcal{X}, \sqsubseteq)$ is a partially ordered set. Moreover for any increasing sequence $\left\{x_{n}\right\}$ in $\mathcal{X}$ converging to $x^{*} \in \mathcal{X}$, we have $x_{n}(t) \sqsubseteq x^{*}(t)$ for any $t \in W$. Hence, the condition (vi) of Theorem 3.2 in $\left(\mathcal{X},\|\cdot\|_{\infty}, \sqsubseteq\right)$ is fulfilled.

In this section, we study the existence and uniqueness of a common solution of the following functional equations arising in dynamic programming:

$$
q(x)=\sup _{y \in \mathcal{D}}\left\{\mathcal{H}_{i}(x, y, q(\tau(x, y)))\right\}, \quad x \in W, \quad i \in\{1,2,3,4\} .
$$

Consider the operators $\Im_{i}: \mathcal{X} \rightarrow \mathcal{X}$ given by

$$
\Im_{i} h(x)=\sup _{y \in \mathcal{D}}\left\{\mathcal{H}_{i}(x, y, h(\tau(x, y)))\right\},
$$

for $h \in \mathcal{X}, x \in W$, where $i \in\{1,2,3,4\}$; these mappings are well-defined if the functions $\mathcal{H}_{i}$ are bounded.

Theorem 4.1. Let $\Im_{i}: \mathcal{X} \rightarrow \mathcal{X}$ be given by (4.2), where $i \in\{1,2,3,4\}$. Suppose that the following hypotheses hold:

(D1) $\mathcal{H}_{i}: W \times \mathcal{D} \times \mathbb{R} \rightarrow \mathbb{R}$ are bounded functions, where $i \in\{1,2,3,4\}$;

(D2) There exists $\lambda \geq 0$ such that, for all $x \in W, y \in \mathcal{D}$ and $\ell_{i}, \hbar_{i} \in \mathbb{R}$,

$$
\left|\mathcal{H}_{i}\left(x, y, \ell_{i}\right)-\mathcal{H}_{i}\left(x, y, \hbar_{i}\right)\right| \leq \lambda\left|\ell_{i}-\hbar_{i}\right| \quad \text { for all } i=1,2,3,4 .
$$

(D3) for all $t \in W, s \in \mathcal{D}, h \in \mathcal{X}$, we have:

$$
h(t) \leq \mathcal{H}_{1}(t, s, h(s)) \text { and } h(t) \leq \mathcal{H}_{2}(t, s, h(s)) ;
$$

(D4) for all $(t, s) \in W \times \mathcal{D}, \varsigma \in W h \in \mathcal{X}$, we have:

$$
\mathcal{H}_{3}(t, s, h(\varsigma)) \leq \mathcal{H}_{1}\left(t, s, \mathcal{H}_{3}(s, \tau, h(\varsigma))\right), \quad \mathcal{H}_{4}(t, s, h(\varsigma)) \leq \mathcal{H}_{2}\left(t, s, \mathcal{H}_{4}(s, \tau, h(\varsigma))\right) ;
$$

(D5) for all $t \in W, s \in \mathcal{D}, h \in \mathcal{X}$, we have:

$$
\mathcal{H}_{1}\left(t, s, \mathcal{H}_{3}(s, \tau, h(\tau))\right) \leq h(t), \quad \mathcal{H}_{2}\left(t, s, \mathcal{H}_{4}(s, \tau, h(\tau))\right) \leq h(t)
$$

$$
\left\{\begin{array}{l}
\text { for all } t \in W, h \in \mathcal{X}, \Im_{1} \Im_{4} h(t)=\Im_{4} \Im_{1} h(t), \text { whenever } \Im_{1} h(t)=\Im_{4} h(t), \text { and } \\
\left\{\begin{array}{l}
\text { there exists }\left\{k_{n}\right\} \subset \mathcal{X} \text { such that } \lim _{n \rightarrow \infty} \Im_{2} k_{n}=\lim _{n \rightarrow \infty} \Im_{3} k_{n}=k^{*} \in \mathcal{X} \\
\text { and } \lim _{n \rightarrow \infty} \sup _{x \in W}\left|\Im_{2} \Im_{3} k_{n}-\Im_{3} \Im_{2} k_{n}\right|=0
\end{array}\right.
\end{array}\right.
$$

$$
\text { or }
$$

$\left\{\begin{array}{l}\text { for all } t \in W, h \in \mathcal{X}, \Im_{2} \Im_{3} h(t)=\Im_{3} \Im_{2} h(t), \text { whenever } \Im_{2} h(t)=\Im_{3} h(t), \text { and } \\ \left\{\begin{array}{l}\text { there exists }\left\{h_{n}\right\} \subset \mathcal{X} \text { such that } \lim _{n \rightarrow \infty} \Im_{1} h_{n}=\lim _{n \rightarrow \infty} \Im_{4} h_{n}=h^{*} \in \mathcal{X} \\ \text { and } \lim _{n \rightarrow \infty} \sup _{x \in W}\left|\Im_{1} \Im_{4} h_{n}-\Im_{4} \Im_{1} h_{n}\right|=0 ;\end{array}\right.\end{array}\right.$ 
(D7) the functions $\mathcal{H}_{i}: W \times \mathcal{D} \times \mathbb{R} \rightarrow \mathbb{R}, i \in\{1,2,3,4\}$, satisfy

$$
\begin{aligned}
& \left|\mathcal{H}_{1}(x, y, h(x))-\mathcal{H}_{2}(x, y, k(x))\right| \\
& \leq a \max \left\{\begin{array}{l}
\left.\left|\Im_{4} h(s)-\Im_{3} k(s)\right|\right),\left|\Im_{3} h(s)-\Im_{1} h(s)\right|,\left|\Im_{4} k(s)-\Im_{2} k(s)\right|, \\
\frac{1}{2}\left[\left|\Im_{4} h(s)-\Im_{2} k(s)\right|+\left|\Im_{3} k(s)-\Im_{1} h(s)\right|\right]
\end{array}\right\} \\
& +b \max \left\{\left|\Im_{4} h(s)-\Im_{1} h(s)\right|,\left|\Im_{3} k(s)-\Im_{2} h(s)\right|,\right\} \\
& +c\left[\left|\Im_{4} h(s)-\Im_{2} k(s)\right|+\left|\Im_{3} k(s)-\Im_{1} h(s)\right|\right] \\
& :=R(h(s), k(s))
\end{aligned}
$$

for all $h, k \in \mathcal{X}, s \in W$, and some $0 \leq a, b, c>0$ and $a+b+2 c=1$.

Then the system of functional equations (4.1) has a bounded solution.

Proof. First of all we prove that $\Im_{i} u$ is a bounded function on $W$, that is, $\Im_{i} u \in \mathcal{X}$ and the operators $\Im_{i}$ are well-defined.

We only need to prove that, for all $u \in \mathcal{X}$, the function $\Im_{1} u: W \rightarrow \mathbb{R}$ is bounded. Indeed, let $u \in \mathcal{X}$ be arbitrary. As $u$ is bounded, by hypothesis $(D 1)$, there exists $\lambda_{1}>0$ such that

$$
|u(x)| \leq \lambda_{1} \text { for all } x \in W .
$$

By hypothesis $(D 1)$, there exists $\lambda_{2}>0$ such that, for all $x \in W$ and all $y \in \mathcal{D}$,

$$
\left|\mathcal{H}_{1}(x, y, 0)\right| \leq \lambda_{2} \text {. }
$$

Now by hypothesis (D2), for all $x \in W$ and all $y \in \mathcal{D}$,

$$
\begin{aligned}
\mid \mathcal{H}_{1}(x, y, u(\tau(x, y)) \mid & =\mid \mathcal{H}_{1}\left(x, y, u(\tau(x, y))-\mathcal{H}_{1}(x, y, 0)|+| \mathcal{H}_{1}(x, y, 0) \mid\right. \\
& \leq \lambda \mid u\left(\tau(x, y) \mid+\lambda_{2} \leq \lambda \lambda_{1}+\lambda_{2} .\right.
\end{aligned}
$$

As a result, for all $x \in W$, we have that

$$
\left|\Im_{1} h(x)\right| \leq \sup _{y \in \mathcal{D}}\left|\mathcal{H}_{1}\left(x, y, h_{1}(\tau(x, y))\right)\right| \leq \lambda \lambda_{1}+\lambda_{2}
$$

That implies that $\Im_{1} u$ is a bounded function on $W$, that is, $\Im_{1} u \in \mathcal{X}$ and the operator $\Im_{1}$ is well-defined. Similarly we can show that other $\Im_{i}(i=2,3,4)$ are well-defined.

Now, let $\lambda$ be an arbitrary positive number, $x \in W$ and $h_{1}, h_{2} \in \mathcal{X}$. Then there exist $y_{1}, y_{2} \in \mathcal{D}$ such that

$$
\begin{aligned}
& \Im_{1} h_{1}(x)<\mathcal{H}_{1}\left(x, y_{1}, h_{1}\left(\tau\left(x, y_{1}\right)\right)\right)+\lambda, \\
& \Im_{2} h_{2}(x)<\mathcal{H}_{2}\left(x, y_{2}, h_{2}\left(\tau\left(x, y_{2}\right)\right)\right)+\lambda, \\
& \Im_{1} h_{1}(x) \geq \mathcal{H}_{1}\left(x, y_{2}, h_{1}\left(\tau\left(x, y_{2}\right)\right)\right), \\
& \Im_{2} h_{2}(x) \geq \mathcal{H}_{2}\left(x, y_{1}, h_{2}\left(\tau\left(x, y_{1}\right)\right)\right) .
\end{aligned}
$$

Let $h_{1}, h_{2} \in \mathcal{X}$. Using hypothesis $(D 3),(4.5)$ and (4.6), for all $t \in W$, we have

$$
h_{1}(t) \leq \Im_{1} h_{1}(t) \text { and } h_{2}(t) \leq \Im_{2} h_{2}(t) .
$$

Then we have $h \sqsubseteq \Im_{1} h$ and $h \sqsubseteq \Im_{2} h$ for all $h \in \mathcal{X}$. This implies that $\Im_{1}$ and $\Im_{2}$ are dominating maps. 
Let $h \in \mathcal{X}$. Using hypothesis $(D 4)$, for all $t \in W$, we have

$$
\begin{aligned}
\Im_{3} h(t) & =\sup _{s \in D} \mathcal{H}_{3}(t, s, h(\varsigma)) \leq \sup _{s \in D} \mathcal{H}_{1}\left(t, s, \mathcal{H}_{3}(s, \tau, h(\varsigma))\right) \\
& \leq \sup _{s \in D} \mathcal{H}_{1}\left(t, s, \Im_{3} h(s)\right)=\Im_{1} \Im_{3} h(t) .
\end{aligned}
$$

Similarly, using hypothesis $(D 4)$, for all $t \in W$, we have

$$
\begin{aligned}
\Im_{4} h(t) & =\sup _{s \in D} \mathcal{H}_{4}(t, s, h(\varsigma)) \leq \sup _{s \in D} \mathcal{H}_{2}\left(t, s, \mathcal{H}_{4}(s, \tau, h(\varsigma))\right) \\
& \leq \sup _{s \in D} \mathcal{H}_{2}\left(t, s, \Im_{4} h(s)\right)=\Im_{2} \Im_{4} h(t) .
\end{aligned}
$$

Then, we have $\Im_{3} h \sqsubseteq \Im_{1} \Im_{3} h$ and $\Im_{4} h \sqsubseteq \Im_{2} \Im_{4} h$ for all $h \in W$. This implies that the pairs $\left(\Im_{3}, \Im_{1}\right)$ and $\left(\Im_{4}, \Im_{2}\right)$ are partially weakly increasing.

Let $h \in \mathcal{X}$. Using hypothesis $(D 5)$, for all $t \in W$, we have

$$
\Im_{1} \Im_{3} h(t) \leq h(t) \text { and } \Im_{2} \Im_{4} h(t) \leq h(t) .
$$

Then, we have $\Im_{1} \Im_{3} h \sqsubseteq h$ and $\Im_{2} \Im_{4} h \sqsubseteq h$ for all $h \in \mathcal{X}$. This implies that $\Im_{1}$ and $\Im_{2}$ are weak annihilators of $\Im_{3}$ and $\Im_{4}$ respectively.

From hypothesis $(D 6)$, the pair $\left(\Im_{1}, \Im_{4}\right)$ is weakly compatible and $\left(\Im_{2}, \Im_{3}\right)$ is compatible, or the pair $\left(\Im_{2}, \Im_{3}\right)$ is weakly compatible and $\left(\Im_{1}, \Im_{4}\right)$ is compatible.

Now, by using (4.3), (4.6) and hypothesis (D7), we obtain

$$
\begin{aligned}
\Im_{1} h_{1}(x)-\Im_{1} h_{2}(x) & <\mathcal{H}_{1}\left(x, y_{1}, h_{1}\left(\tau\left(x, y_{1}\right)\right)\right)-\mathcal{H}_{2}\left(x, y_{1}, h_{2}\left(\tau\left(x, y_{1}\right)\right)\right)+\lambda \\
& \leq\left|\mathcal{H}_{1}\left(x, y_{1}, h_{1}\left(\tau\left(x, y_{1}\right)\right)\right)-\mathcal{H}_{2}\left(x, y_{1}, h_{2}\left(\tau\left(x, y_{1}\right)\right)\right)\right|+\lambda \\
& \leq R\left(h_{1}(x), h_{2}(x)\right)+\lambda
\end{aligned}
$$

and so we have

$$
\Im_{1} h_{1}(x)-\Im_{2} h_{2}(x)<R\left(h_{1}(x), h_{2}(x)\right)+\lambda .
$$

Analogously, by using (4.4) and (4.5), we get

$$
\Im_{1} h_{2}(x)-\Im_{1} h_{1}(x)<R\left(h_{1}(x), h_{2}(x)\right)+\lambda
$$

Finally, from (4.7) and (4.8), we deduce

$$
\left|\Im_{1} h_{1}(x)-\Im_{2} h_{2}(x)\right|<R\left(h_{1}(x), h_{2}(x)\right)+\lambda,
$$

implying that

$$
d_{\infty}\left(\Im_{1} h_{1}, \Im_{2} h_{2}\right) \leq R\left(h_{1}, h_{2}\right)+\lambda .
$$

Notice that the last inequality does not depend on $x \in W$ and $\lambda>0$ is taken arbitrarily, therefore we obtain that

$$
\begin{aligned}
& d_{\infty}\left(\Im_{1} h_{1}, \Im_{2} h_{2}\right) \\
& \leq a \max \left\{\begin{array}{l}
d_{\infty}\left(\Im_{4} h(s), \Im_{3} k(s)\right), d_{\infty}\left(\Im_{3} h(s), \Im_{1} h(s)\right), d_{\infty}\left(\Im_{4} k(s), \Im_{2} k(s)\right), \\
\frac{1}{2}\left[d_{\infty}\left(\Im_{4} h(s), \Im_{2} k(s)\right)+d_{\infty}\left(\Im_{3} k(s), \Im_{1} h(s)\right)\right]
\end{array}\right\} \\
& \quad+b \max \left\{d_{\infty}\left(\Im_{4} h(s), \Im_{1} h(s)\right), d_{\infty}\left(\Im_{3} k(s), \Im_{2} h(s)\right)\right\} \\
& \quad+c\left[d_{\infty}\left(\Im_{4} h(s), \Im_{2} k(s)\right)+d_{\infty}\left(\Im_{3} k(s), \Im_{1} h(s)\right)\right] .
\end{aligned}
$$

Hence Theorem 3.2 is applicable since all its hypotheses are satisfied for operators $\mathcal{A}=\Im_{1}, \mathcal{B}=\Im_{2}, \Im=\Im_{3}$ and $\mathcal{S}=\Im_{4}$. Thus, there exists a common fixed point of 
$\mathcal{A}, \mathcal{B}, \mathcal{S}$ and $\Im$, i.e., a bounded solution $\nu^{*} \in \mathcal{X}$ such that $\Im_{i} \nu^{*}=\nu^{*}$. In other words, for all $x \in W$,

$$
\nu^{*}(x)=\Im_{i} \nu^{*}(x)=\sup _{y \in \mathcal{D}}\left\{\mathcal{H}_{i}\left(x, y, \nu^{*}(\tau(x, y))\right)\right\} .
$$

This completes the proof.

Acknowledgements. The first author is thankful to the United State-India Education Foundation, New Delhi, India and IIE/CIES, Washington, DC, USA on selection for Fulbright-Nehru PDF Award (No. 2052/FNPDR/2015).

\section{References}

[1] Abbas, M., Nazir, T., Radenović, S., Common fixed point of four maps in partially ordered metric spaces, Appl. Math. Lett., 24(2011), 1520-1526.

[2] Agarwal, R.P., El-Gebeily, M.A., O'Regan, D., Generalized contractions in partially ordered metric spaces, Appl. Anal., 87(2008), 1-8.

[3] Altun, I., Simsek, H., Some fixed point theorems on ordered metric spaces and application, Fixed Point Theory Appl., 2010 (2010), Article ID 621492, 17 pages.

[4] Banach, S., Sur les operations dans les ensembles abstraits et leur application aux equations integerales, Fund. Math., 3(1922), 133-181.

[5] Bellman, R., Lee, E. S., Functional equations in dynamic programming, Aequationes Math., 17(1978), 1-18.

[6] Bhakta, P.C., Mitra, S., Some existence theorems for functional equations arising in dynamic programming, J. Math. Anal. Appl., 98(1984), no. 2, 348-362.

[7] Browder, F.E., Petryshyn, W.V., Construction of fixed points of nonlinear mappings in Hilbert spaces, J. Math. Anal. Appl., 20(1967), 197-228.

[8] Bruck, R.E., Asymptotic behaviour of nonexpansive mappings, Contemporary Mathematics 18, Fixed Points and Nonexpansive Mappings, (R.C. Sine, Ed.), AMS, Providence, RI, 1980.

[9] Ćirić, Lj. B., A generalization of Banach's contraction principle, Proc. Am. Math. Soc., 45(1974), 267-273.

[10] Ćirić, Lj. B., On some nonexpansive mappings and fixed points, Indian J. Pure Appl. Math., 24(1993), no. 3, 145-149.

[11] Ćirić, Lj. B. A new class of nonexpansive type mappings and fixed points, Czech. Math. J., 49(124)(1999), 891-899.

[12] Ding, H.Sh., Nashine, H.K, Kadelburg, Z., Common fixed point theorems for weakly increasing mappings on ordered orbitally complete metric spaces, Fixed Point Theory Appl., 85(2012), 14 pages.

[13] Harjani, J., Sadarangani, K., Fixed point theorems for weakly contractive mappings in partially ordered sets, Nonlinear Anal., 71(2009), 3403-3410.

[14] Kirk, W.A., Fixed point theorems for nonexpansive mappings satisfying certain boundary conditions, Proc. Amer. Math. Soc., 50(1975), 143-149.

[15] Matkowski, J., Integrable solutions of functional equations, Dissertationes Math., 127(1975), 1-68.

[16] Matkowski, J., Fixed point theorems for mappings with a contractive iterate at a point, Proc. Amer. Math. Soc., 62(1977), 344-348. 
[17] Nashine, H.K., Kadelburg, Z., Generalized nonexpansive mappings in ordered metric spaces and their (common) fixed points with applications, RACSAM, 108(2014), 633651.

[18] Nashine, H.K., Kadelburg, Z., Implicit relations related to ordered orbitally complete metric spaces and applications, RACSAM, 111(2017), 403-424.

[19] Nashine, H.K., Kadelburg, Z., Golubović, Z., Common fixed point results using generalized altering distances on orbitally complete ordered metric spaces, J. Appl. Math., 2012, Article ID 382094, 1-13.

[20] Nashine, H.K., Samet, B., Fixed point results for mappings satisfying $(\psi, \varphi)$-weakly contractive condition in partially ordered metric spaces, Nonlinear Anal. TMA, 74(2011), 2201-2209.

[21] Nieto, J.J., López, R.R., Contractive mapping theorems in partially ordered sets and applications to ordinary differential equations, Order, 22(2005), 223-239.

[22] O'Regan, D., Petruşel, A., Fixed point theorems for generalized contractions in ordered metric spaces. J. Math. Anal. Appl., 341(2008), 1241-1252.

[23] Parvaneh, V., Razani, A., Roshan, J.R., Common fixed points of six mappings in partially ordered G-metric spaces, Math. Sci., 7(2013):18.

[24] Pathak, H.K., Cho, Y.J., Kang, S.M., Lee, B.S., Fixed point theorems for compatible mappings of type $(P)$ and applications to dynamic programming, Le Mathematiche, 50(1995), no. 1, 15-33.

[25] Petrusel, A., Rus, I.A., Fixed point theorems in ordered L-spaces, Proc. Amer. Math. Soc., 134(2006), 411-418.

[26] Ran, A.C.M., Reurings, M.C.B., A fixed point theorem in partially ordered sets and some applications to matrix equations, Proc. Amer. Math. Soc., 132(2004), 1435-1443.

[27] Reich, S., Fixed points of nonexpansive functions, J. London Math. Soc., 7(1973), no. $2,5-10$.

[28] Sastry, K.P.R., Naidu, S.V.R., Rao, I.H.N., Rao, K.P.R., Common fixed points for asymptotically regular mappings, Indian J. Pure Appl. Math., 15(1984), 849-854.

[29] Shatanawi, W., Samet, B., On $(\psi, \phi)$-weakly contractive condition in partially ordered metric spaces, Comput. Math. Appl., 62(2011), 3204-3214.

[30] Singh, S.L., Mishra, S.N., On Ljubomir Ćirić fixed point theorem for nonexpansive type maps with applications, Indian J. Pure Appl. Math., 33(2002), no. 4, 531-542.

[31] Turinici, M., Abstract comparison principles and multivariable Gronwall-Bellman inequalities, J. Math. Anal. Appl., 117(1986), 100-127.

[32] Turinici, M., Fixed points for monotone iteratively local contractions, Demonstratio Math., 19(1986), 171-180.

Hemant Kumar Nashine

Texas A \& M University - Kingsville

Department of Mathematics

78363-8202, Texas, USA

e-mail: drhknashine@gmail.com 
Ravi P. Agarwal

Texas A \& M University - Kingsville

Department of Mathematics

78363-8202, Texas, USA

e-mail: agarwal@tamuk.edu 